\title{
The Effect of Filter Dimension on the Subgrid-Scale Stress, Heat Flux, and Tensor Alignments in the Atmospheric Surface Layer
}

\author{
Chad W. Higgins \\ Center for Environmental and Applied Fluid Mechanics, and Department of Geography and Environmental Engineering, The Johns \\ Hopkins University, Baltimore, Maryland \\ Charles Meneveau \\ Center for Environmental and Applied Fluid Mechanics, and Department of Mechanical Engineering, The Johns Hopkins University, \\ Baltimore, Maryland \\ Marc B. PARLANGE \\ Center for Environmental and Applied Fluid Mechanics, and Department of Geography and Environmental Engineering, The Johns \\ Hopkins University, Baltimore, Maryland, and School of Architecture, Civil and Environmental Engineering, Ecole Polytechnique \\ Fédérale de Lausanne, Lausanne, Switzerland
}

(Manuscript received 27 December 2005, in final form 24 July 2006)

\begin{abstract}
In field experiments designed to study subgrid-scale parameterizations for large eddy simulation, the flow field is often measured and then filtered in two-dimensional planes. This two-dimensional filtering serves as a surrogate for three-dimensional filtering. The question of whether this will yield accurate results in subgrid-scale (SGS) models is addressed by analyzing data from a field experiment in which 16 sonic anemometers were deployed in a four by four grid. The experiment was held in July 2002 at the Surface Layer Turbulence and Environmental Science Test (SLTEST) facility in the Utah West Desert. The full SGS stress tensor and its parameterizations using both two- and three-dimensional filterings are obtained. Comparisons are given between two- and three-dimensional filterings of the field measurements based on probability density functions (PDFs) and energy spectra of the SGS stress elements. The PDFs reveal that quantities calculated with two-dimensional filtering exhibit greater intermittency than those computed with three-dimensional filtering at the same scale. From the spectra it is observed that the different filtering methods result in similar behavior, but that spectra of SGS stress components computed with a threedimensional filter roll off at a slightly lower wavenumber than those computed with a two-dimensional filter. The PDFs and spectra of the stresses calculated with two- and three-dimensional filters can be made to collapse by reducing the three-dimensional filter scale according to $\Delta_{3-D}=0.84 \Delta_{2-D}$. Geometric alignment analyses are performed for the SGS heat flux, SGS stress, and filtered strain rate for the cases of stable, near-neutral, and unstable atmospheric stabilities. Under unstable and near-neutral atmospheric stability, two-dimensional filtering yields acceptable results; however, under stable atmospheric stability, a new approach is recommended and delineated.
\end{abstract}

\section{Introduction}

To investigate the characteristics of subgridscale (SGS) variables and to evaluate closure models for large eddy simulation (LES), several field ex-

Corresponding author address: Chad Higgins, The Johns Hopkins University, 313 Ames Hall, 3400 N. Charles St., Baltimore, MD 21218.

E-mail: chad@jhu.edu

DOI: 10.1175/JTECH1991.1

(C) 2007 American Meteorological Society periments have recently been performed in the atmospheric surface layer using various horizontal arrays of sonic anemometers (Tong et al. 1999, 1998; Porté-Agel et al. 2000a,b, 2001a,b; Higgins et al. 2003, 2004; Horst et al. 2004; Kleissl et al. 2003, 2004; Sullivan et al. 2003). In these experiments, two-dimensional filtering was used to compute the components of the SGS stress tensor. However, the LES equations are derived using a three-dimensional filter: 


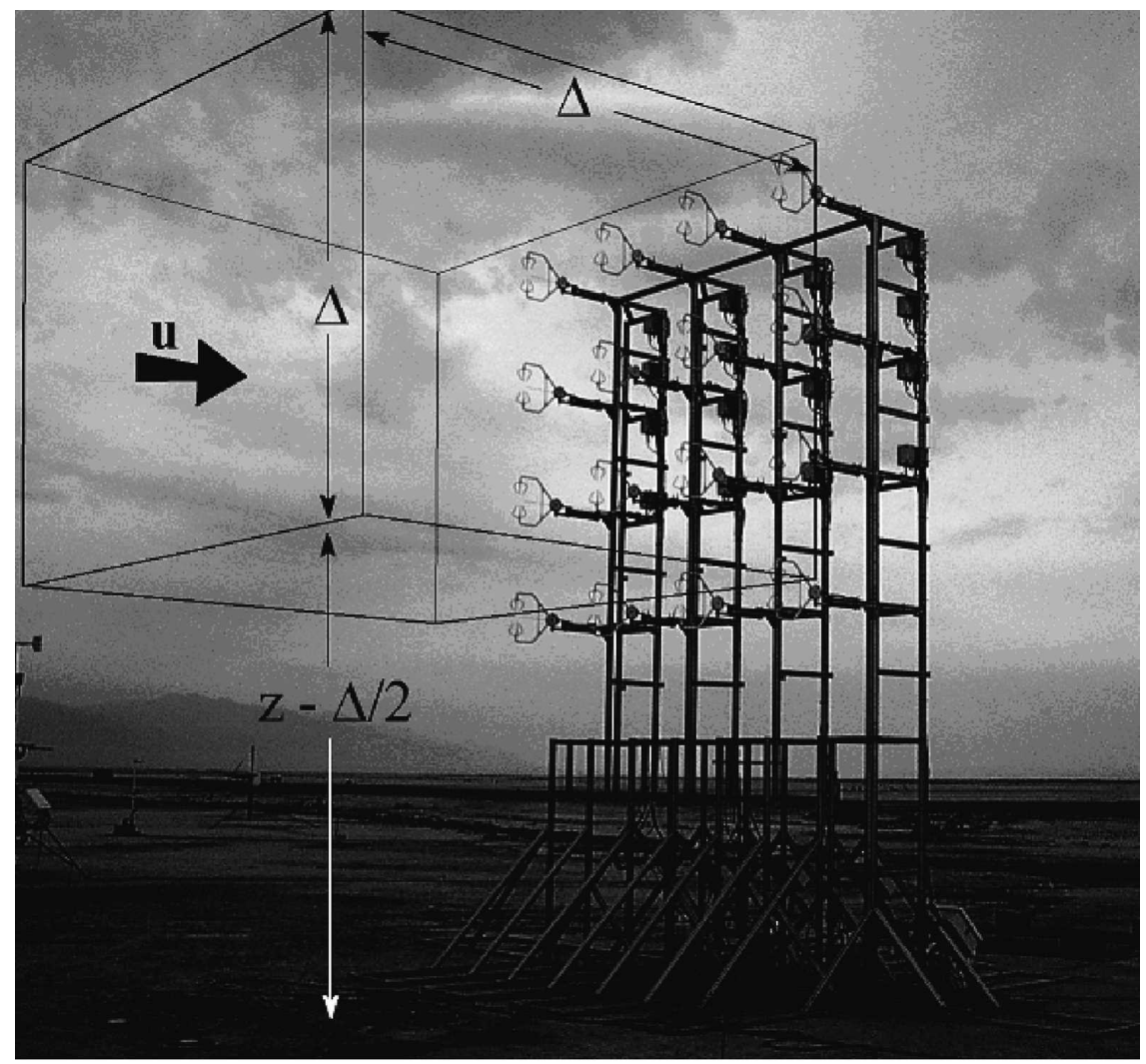

FIG. 1. Photograph of the SGS 2002 experiment. Sixteen sonic anemometers were arranged in a $4 \times 4$ grid. A sample three-dimensional filter domain is indicated as the box. The array of anemometers was orientated perpendicular to the mean wind direction and measurements of the temperature and the three components of the velocity vector were logged at $20 \mathrm{~Hz}$.

$$
\frac{\partial \tilde{u}_{i}}{\partial t}+\tilde{u}_{j} \frac{\partial \tilde{u}_{i}}{\partial x_{j}}=-\frac{1}{\rho} \frac{\partial \tilde{p}}{\partial x_{i}}+\nu \frac{\partial^{2} \tilde{u}_{i}}{\partial x_{j}^{2}}-\frac{\partial}{\partial x_{j}} \tau_{i j}
$$

where $\tilde{u}_{i}$ is the filtered velocity field, $\tilde{p}$ is the filtered pressure, $\nu$ is the kinematic viscosity, $\rho$ is the density, and $\tau_{i j}={\overline{u_{i}}}_{j}-\tilde{u}_{i} \tilde{u}_{j}$ is the unknown SGS stress. Here, the tilde $(\sim)$ represents spatial filtering. The assumption inherent in all the analyses of the field data is that two-dimensional filtering is an appropriate surrogate for the three-dimensional filtering that is applied to Navier-Stokes equations to derive the LES equation set. Tong et al. (1998) addressed this issue using LES of the atmospheric boundary layer, and showed that the reduction in filter dimension increased the variance of the LES variables by $10 \%-14 \%$. Here, we extend the analysis of Tong et al. using field data from a 2002 field campaign in Utah that was designed to allow for threedimensional filtering. The specific correspondence between a particular filter type and the implicit filter in an actual numerical simulation has been a much-discussed topic (see, e.g., Piomelli et al 1988; De Stefano and Vasilyev 2002) that is beyond the scope of the present paper.
In this paper we compare the probability density functions and the spectra of the components of the SGS stress and filtered strain rate with two-dimensional versus three-dimensional filtering. A vector alignment analysis of the SGS heat flux is performed for both twoand three-dimensional filterings, and a tensor alignment analysis of the SGS stress and filtered strain rate is performed for both two- and three-dimensional filterings. We confirm the increase in variability discussed by Tong et al., and identify that this increase in variability associated with two-dimensional filtering can be interpreted as a $16 \%$ reduction in the associated threedimensional filter size. We use a geometric analysis to

TABLE 1. Data characterization: one segment corresponds to $\sim 30$ min of measurements. Taylor's hypothesis is used to convert the temporal data into streamwise data.

\begin{tabular}{lccc}
\hline \hline & Stable & Neutral & Unstable \\
\hline$(z / L)$ & $0.15>(z / L)$ & $|(z / L)|$ & $-1.6<(z / L)$ \\
& $>0.05$ & $<0.03$ & $<-0.05$ \\
No. of segments & 4 & 34 & 28 \\
$\sim$ No. of points & 150000 & 1200000 & 1000000 \\
\hline
\end{tabular}



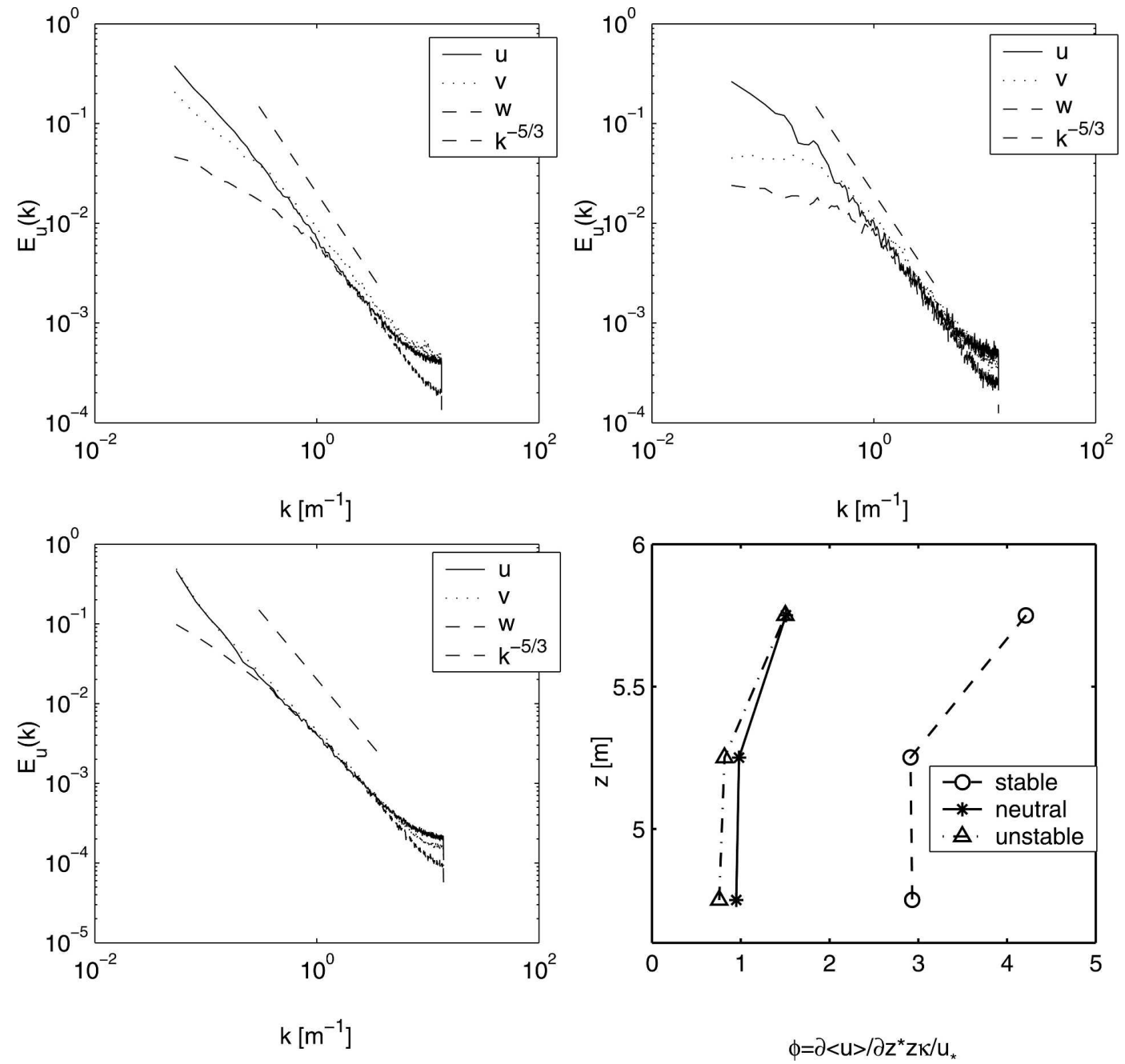

FIG. 2. Spectra of $u$, $v$, and $w$, for the atmosphere under (a) neutral, (b) stable, and (c) unstable stabilities. The spectra show the expected $k^{-5 / 3}$ slope. (d) Normalized average velocity gradients for all three cases of atmospheric stability.

show that, for the case of stably stratified flow, twodimensional filtering can give incorrect results. We make recommendations on data processing and the directionality of the filtering operations.

\section{SGS 2002 field experment}

Sixteen sonic anemometers were deployed at the Surface Layer Turbulence and Environmental Science Test (SLTEST) site in the West Desert of Utah from 8 to 23 July 2002. The anemometers were arranged in a $4 \times 4$ grid perpendicular to the mean wind direction. The array center was placed $5.25 \mathrm{~m}$ above the ground, with a horizontal and vertical spacing between the sonics of $\sim 0.5 \mathrm{~m}$ resulting in a filter size $(\Delta)$ of $2 \mathrm{~m}$. The upwind fetch in the West Desert was $\sim O(100 \mathrm{~km})$. A photograph of the experimental setup is presented in
Fig. 1. Measurements of the temperature and of the three components of the velocity vector were logged at $20 \mathrm{~Hz}$. The parameter $z / L$ was used to classify the data into three stability regimes, where $\mathrm{z}$ is the mean instrument height $(5.25 \mathrm{~m}), L$ is the Obukhov length $L=$ $-u_{*}^{3} T_{a} \rho c_{p} / \kappa g H, u_{*}=\left(\left\langle u^{\prime} w^{\prime}\right\rangle^{2}+\left\langle v^{\prime} w^{\prime}\right\rangle^{2}\right)^{(1 / 4)}$ is the friction velocity, $H=\rho c_{p}\left\langle w^{\prime} T^{\prime}\right\rangle$ is the sensible heat flux, $\rho$ is the density of air, $c_{p}$ is the specific heat of air, $T_{a}$ is the reference air temperature, $g$ is the acceleration of gravity, and $\kappa=0.4$ is von Kármán's constant. Table 1 summarizes the stability criteria and resulting amount of data available for analysis. Spectra of the data for all three cases of atmospheric stability are presented in Fig. 2, along with a plot of the average normalized vertical velocity gradients. Note that in the stable case (Fig. 2b) the spectra flatten at small wavenumbers, and the average velocity gradients (Fig. 2d) in the stable case 
are $\sim 3$ times stronger than both the neutral and unstable cases.

In the analysis of SGS stress components, threedimensional filtering is done at a scale of $\Delta=2 \mathrm{~m}$ in all three dimensions by applying a box filter in the vertical and cross-stream horizontal directions. Box filtering is done by performing a weighted average of the sonic outputs in each of the cross-stream directions. Note that the filter size, $\Delta$, need not coincide with the grid spacing. For $\Delta=2 \mathrm{~m}$ the weights are $w_{i}=1 / 4(i=1,2,3$, $4)$, for $\Delta=1.5 \mathrm{~m} w_{i}=1 / 3(i=1,2,3)$, and in the case of $\Delta_{3-D}=0.84 \Delta_{2-D}=1.68 \mathrm{~m}$ the weights are $w_{i}=$ $[0.21,0.29,0.29,0.21]$ across the array. Their weights have been determined by the trapezoidal rule. A Gaussian filter is applied in the streamwise direction. Twodimensional filtered signals are produced by selecting a slice of the data parallel to the land surface at a height of $5.5 \mathrm{~m}$. This slice is filtered with a box filter in the cross-stream horizontal direction with weights $w_{i}=1 / 4$ $(i=1,2,3,4)$ in the $\Delta=2 \mathrm{~m}$ case, and for $\Delta=1.5 \mathrm{~m}$ $w_{i}=1 / 3(i=1,2,3)$. Again, a Gaussian filter is applied in the streamwise direction. There is a small height difference between the 2D and 3D filter locations, which is unavoidable due to array geometry. We have compared the $2 \mathrm{D}$ filtered results for the plane above and below the centroid of the 3D filtered array, and see essentially no difference between the 2D filtered signals above and below the 3D filter centroid. Note that both two- and three-dimensional filterings are done at the same scale $(\Delta=2 \mathrm{~m})$. In the vector and tensor analysis the filter size is reduced to $\Delta=1.5 \mathrm{~m}$ in both the twoand three-dimensional cases to allow for gradients and the calculation of the filtered strain rate, $\tilde{S}_{i j}$.

\section{SGS stress and filtered strain rate components}

We present probability density functions and spectra of the off-diagonal components of the SGS stress tensor, $\tau_{i j}$, and filtered strain rate, $\tilde{S}_{i j}=1 / 2\left(\partial_{i} u_{j}+\partial_{j} u_{i}\right)$. The remaining components show similar behavior with respect to two- and three-dimensional filterings and are not shown. Figures $3 \mathrm{a}-\mathrm{c}$, respectively, present probability density functions of the off-diagonal components of the SGS stress $\tau_{12}, \tau_{13}$, and $\tau_{23}$. In Fig. 3, the dashed

FIG. 3. Probability density functions of the components of the SGS stress tensor calculated with two-dimensional filtering (dashed line) and three-dimensional filtering (solid line) for (a) $\tau_{12}$, (b) $\tau_{13}$, and (c) $\tau_{23}$. In each of the plots, the components computed with two-dimensional filtering exhibit greater intermittency, indicated by the higher probability of extreme events (higher tails) in the PDF.
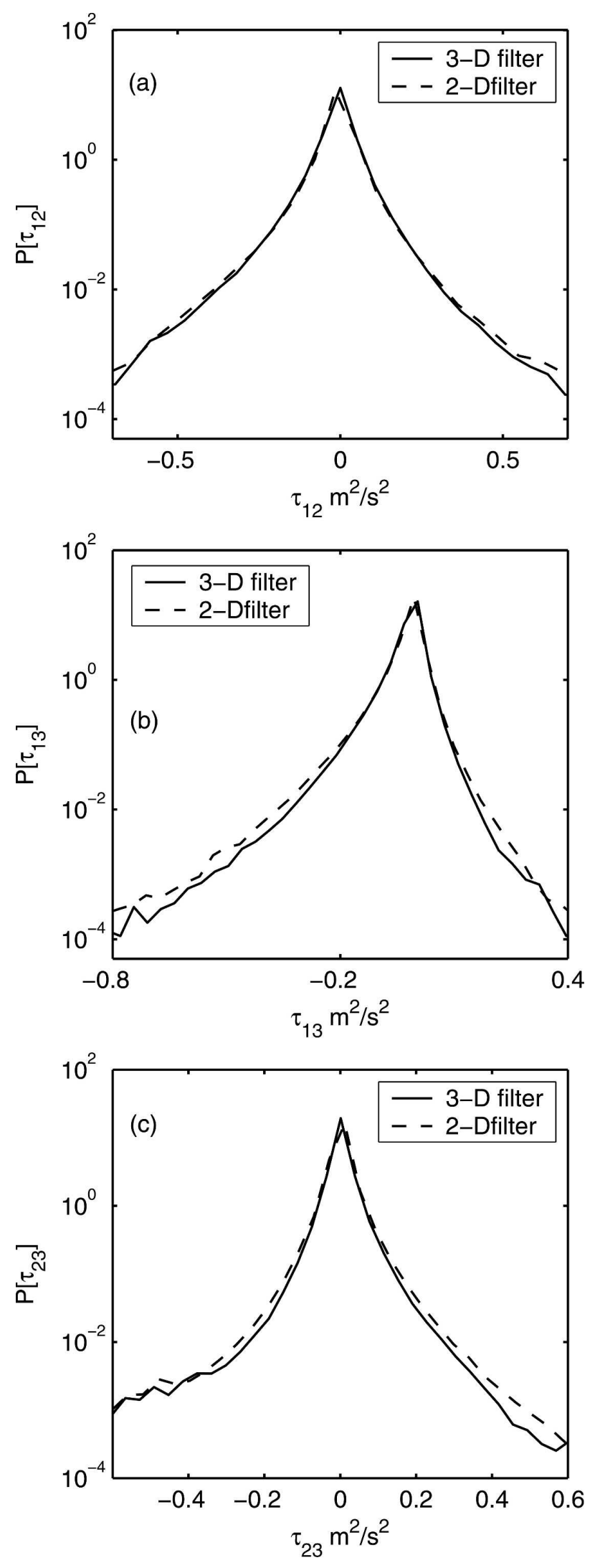
line represents the two-dimensional filtered estimate, and the solid line represents the component calculated with a three-dimensional filter. For all three components, the two-dimensional filtered estimate has larger tails in the PDF indicating a greater degree of intermittency, in agreement with the findings of Tong et al. (1998) in their analysis of LES data. The same trend, increased intermittency in the SGS stress components, was seen using the box filter in all directions (not shown).

In Fig. 4 the corresponding spectra for each offdiagonal component of the SGS stress are presented. Note that each component of the SGS stress computed with three-dimensional filtering (solid lines) has a lower rolloff wavenumber than the corresponding component calculated with two-dimensional filtering (dashed lines). The filter wavenumber, $\pi / \Delta$, is represented by the vertical dotted line. The difference in rolloff wavenumber can be interpreted as the two filters acting on slightly different scales (i.e., the SGS stress components calculated with the three-dimensional filter have fewer small-scale, high-wavenumber structures). To quantify this difference, we ask whether three-dimensional filtering at a scale smaller than the original filter scale $\Delta=2.0 \mathrm{~m}$ would yield similar results to the twodimensional filter. We rescale the filter size such that the spectra of the components of the SGS stress calculated with both two- and three-dimensional filterings collapse. By trial and error we find that rescaling the three-dimensional filter width to $\Delta_{3-D}=0.84 \Delta_{2-D}$ results in a very good collapse, shown in Fig. 5.

The newly identified filter scale is now used to compute the components of the SGS stress with threedimensional filtering at the scale $\Delta_{3-D}=0.84 \Delta_{2-D}$, and a comparison is made to the components calculated with two-dimensional filtering at the original filter scale. This comparison is presented in Fig. 6. The PDFs have now collapsed, showing that the components calculated with two- and three-dimensional filterings have the same level of intermittency when we interpret the three-dimensional filter as one that has a filter size $16 \%$ smaller than the two-dimensional filter. This implies that a two-dimensional filter is a good surrogate for three-dimensional filtering and experiments using two-

FIG. 4. Spectra of the components of the SGS stress tensor calculated with two-dimensional filtering (dashed line) and threedimensional filtering (solid line) for (a) $\tau_{13}$, (b) $\tau_{12}$, and (c) $\tau_{23}$. In each of the plots the components computed with threedimensional filtering have a lower rolloff wavenumber, indicating that the three-dimensional filter is acting on larger scales than the two-dimensional filter.

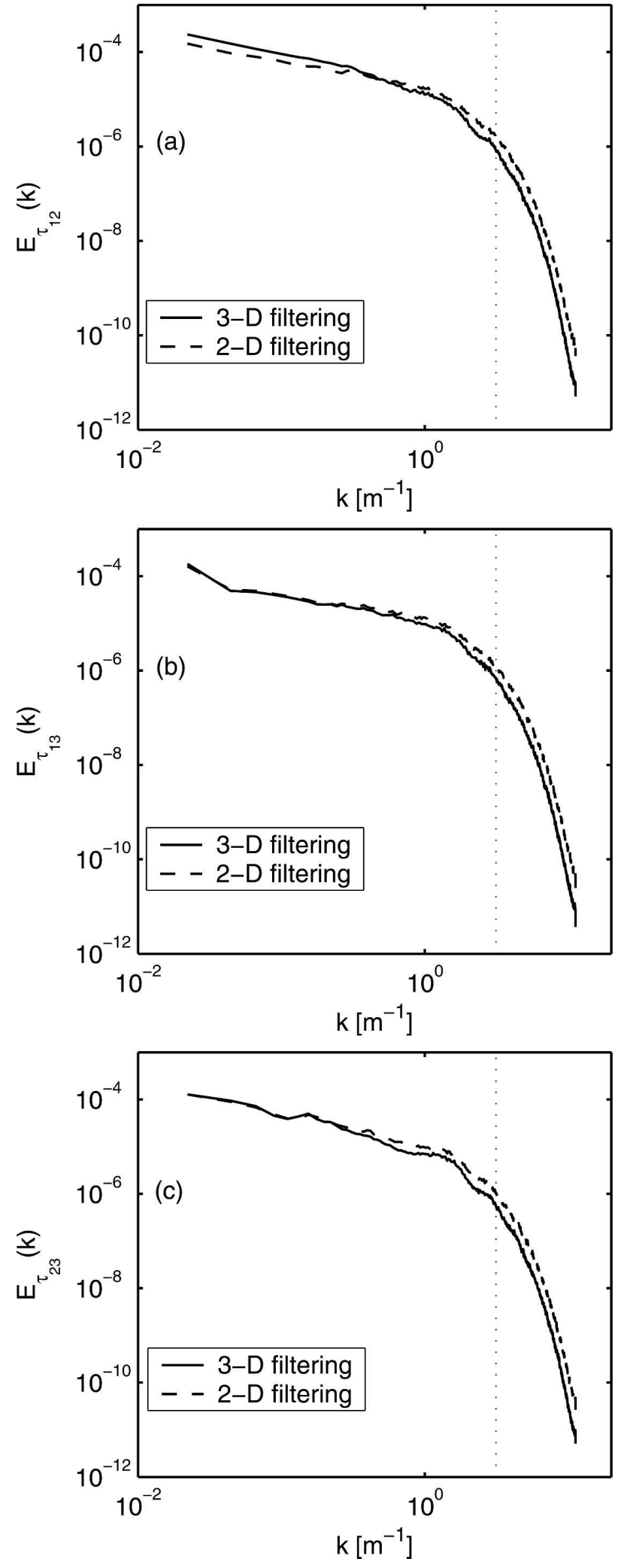



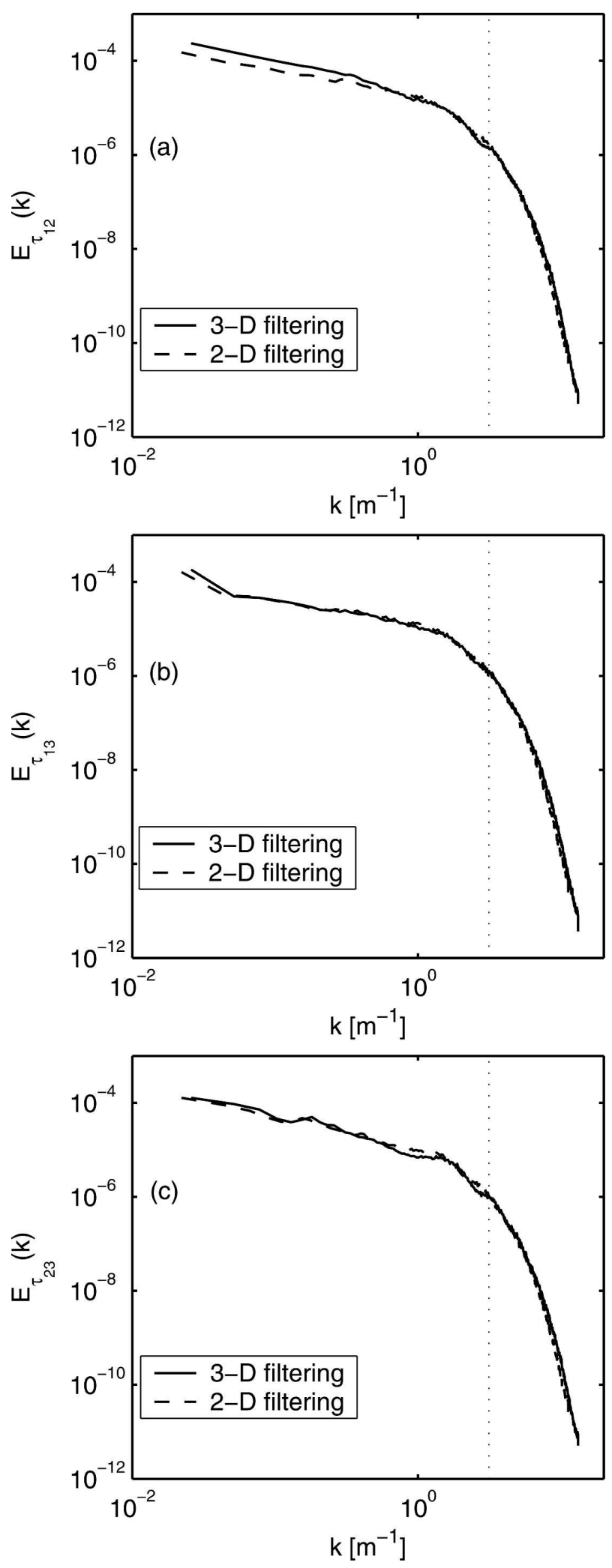

dimensional filtering need only interpret the filter scale properly.

The uncorrected PDFs of $\tilde{S}_{12}, \tilde{S}_{13}$, and $\tilde{S}_{23}$ are shown in Figs. $7 \mathrm{a}-\mathrm{c}$, respectively, while the PDFs of the same components are shown in Figs. 8a-c when $\Delta_{3-D}=$ $0.84 \Delta_{2-D}$. The correction is less dramatic, but an improvement is seen in the collapse of the data. Note that the components of the filtered strain rate have the opposite intermittence behavior as the SGS stress, since filtering causes more attenuation of the large scales while leaving more kinetic energy and variability to the SGS range.

\section{SGS heat flux alignments}

Closure for vector quantities such as the equality of the SGS heat flux and its parameterizations implies that both the direction and magnitude of the SGS flux and its parameterizations must match; that is, the flux and its parameterization must have perfect angular alignment and equal magnitude. The flux of heat (per unit heat capacity) caused by unresolved (SGS) motions occurring at scales smaller than a scale $\Delta$ is defined by

$$
q_{i}(\mathbf{x}, t)=\overline{T u_{i}}-\tilde{T} \tilde{u}_{i}
$$

where $u_{i}$ is the velocity vector, $T$ is the temperature, and the tilde represents spatial filtering at the scale $\Delta$. Here, $\tilde{u}_{i}$ and $\tilde{T}$ are the filtered velocity and temperature fields that are numerically resolved in the LES model. Classical parameterizations for $q_{i}(\mathbf{x}, t)$ in LES are based on down-temperature gradient closures. Specifically, the Smagorinsky (1963) eddy-diffusion parameterization reads

$$
q_{i}(\mathbf{x}, t)=-\frac{c_{s}^{2} \Delta^{2}}{\operatorname{Pr}_{\mathrm{SGS}}}|\tilde{\mathbf{S}}| \frac{\partial T}{\partial x_{i}}\left(\equiv q_{i}^{\text {eddy }}\right),
$$

where $|\tilde{\mathbf{S}}|=\sqrt{2 \tilde{S}_{i j} \tilde{S}_{i j}}$ is the magnitude of the filtered strain rate tensor is the Smagorinsky constant, and $\operatorname{Pr}_{\mathrm{SGS}}$ is the subgrid-scale Prandtl number.

The tensor eddy diffusivity (or nonlinear) model (Leonard 1974; Borue and Orszag 1998) is given by

FIG. 5. Spectra of the SGS stress components computed with two-dimensional (dashed line) and three-dimensional (solid line) filterings at a reduced scale. Here, the wavenumber axis of the components computed with three-dimensional filtering has been rescaled by defining a smaller effective three-dimensional filter size $\Delta_{3-D}=0.84 \Delta_{2-D}$ so that the spectra collapse. 

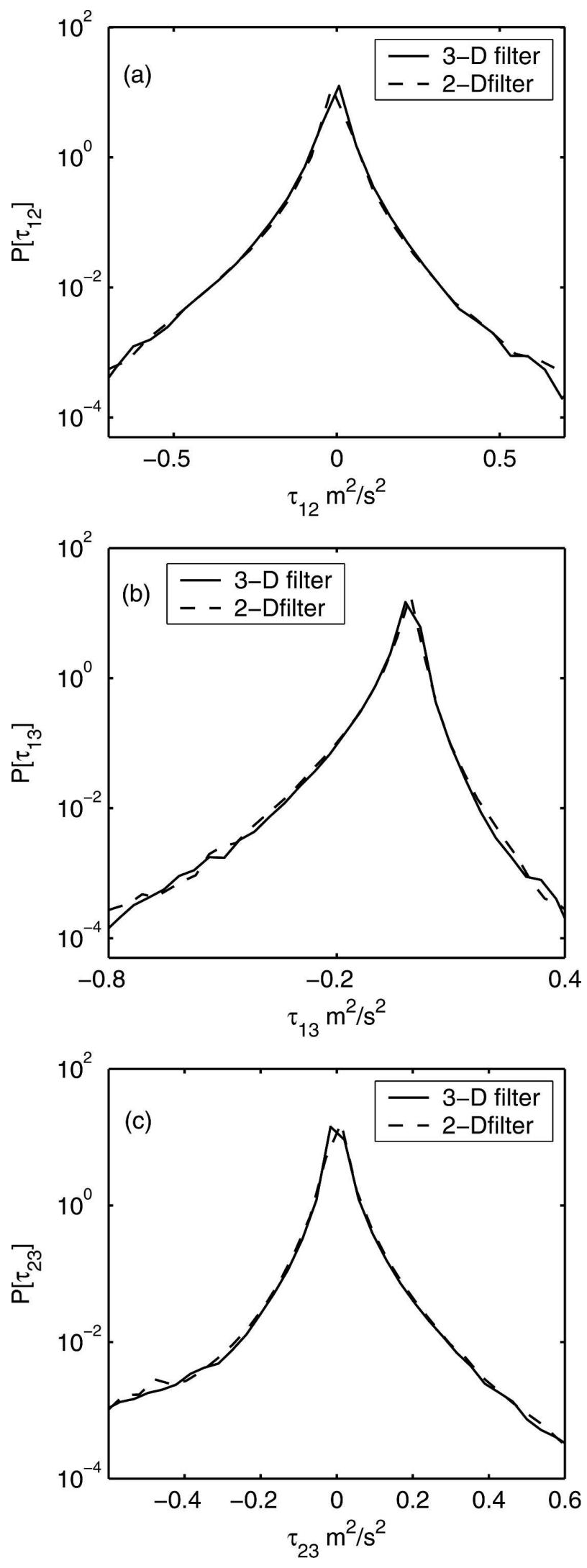

$$
q_{i}(\mathbf{x}, t)=c_{\mathrm{nl}} \Delta^{2} \frac{\partial \tilde{u}_{i}}{\partial x_{k}} \frac{\partial \tilde{T}}{\partial x_{k}}\left(\equiv q_{i}^{\mathrm{nl}}\right),
$$

Where $c_{\mathrm{nl}}$ is the corresponding model coefficient and repeated subscripts are summed over. This can be shown mathematically (Leonard 1997) by keeping the first term in an expansion of the filtered product $\overline{T u_{i}}$ that appears in the definition of $\mathbf{q}$.

A third approach, the so-called mixed model (Bardina and Ferziger 1980), is a linear combination of Eqs. (3) and (4):

$$
q_{i}(\mathbf{x}, t)=-\frac{c_{s}^{2} \Delta^{2}}{\operatorname{Pr}_{T}} \frac{\partial T}{\partial x_{i}}+C_{\mathrm{nl}} \Delta^{2} \frac{\partial u_{i}}{\partial x_{k}} \frac{\partial T}{\partial x_{k}}\left(\equiv q_{i}^{\operatorname{mix}}\right) .
$$

To better understand the relationship between $\mathbf{q}$ and the mixed model when $\mathbf{q}$ is computed with both twoand three-dimensional filterings, we consider the alignments of the real SGS heat flux relative to the "mixed model span," defined as the plane containing the two vectors $\mathbf{q}^{\text {eddy }}$ and $\mathbf{q}^{\text {nl }}$ (this is a well-defined space because these two vectors are highly misaligned). A sketch of the geometry and definition of angles used in this analysis (previously used by Higgins et al. 2004) is presented in Fig. 9. The normal to the mixed model span is given by $\mathbf{q}^{\text {eddy }} \times \mathbf{q}^{\text {nl }}$. The projection, $\mathbf{q}^{\mathbf{p}}$, of the measured heat flux onto the plane spanned by the mixed model is the portion of the SGS heat flux that can be expressed by the mixed model. The location of $\mathbf{q}$ relative to $\mathbf{q}^{\text {eddy }}$ and $\mathbf{q}^{\text {nl }}$ is formally fixed by the angles $\theta=\cos ^{-1}\left(\left(\mathbf{q}^{\text {eddy }} \times \mathbf{q}^{\mathrm{nl}}\right) \cdot \mathbf{q} /\left|\mathbf{q}^{\text {eddy }} \times \mathbf{q}^{\mathrm{n} 1} \| \mathbf{q}\right|\right)$ and $\phi=$ $\cos ^{-1}\left(\mathbf{q}^{\text {eddy }} \cdot \mathbf{q}^{\mathbf{p}} /\left|\mathbf{q}^{\text {eddy }} \| \mathbf{q}^{\mathbf{p}}\right|\right)$. Joint probability density functions (JPDFs) of $\cos (\theta)$ and $\phi$ are computed from the data. The use of $\cos (\theta)$ in the computation of the JPDF ensures that there is no bias toward the $90^{\circ}$ plane. For plotting purposes, this same JPDF is plotted as a function of the spherical angles $\theta$ and $\phi$ on the unit sphere [e.g., random data yields a uniform JPDF in $\cos (\theta)$ and $\phi]$ and would be represented by uniform color on the unit sphere. Figures 10-12 show the measured joint probability density functions of $\theta$ and $\phi$ plotted on the unit sphere for both the two- (shown in panel a) and three-dimensional (shown in panel b) filterings for unstable, near-neutral, and stable datasets, respectively. The joint PDF quantifies the relative frequency of the orientations of the measured SGS heat flux rela-

FIG. 6. PDFs of the SGS stress components computed with two-dimensional filtering (dashed line) and three-dimensional filtering (solid line) at a reduced scale $\Delta_{3-D}=0.84 \Delta_{2-D}$. The difference in intermittency of the signals seen in Fig. 4 has disappeared, and the PDFs have collapsed to one. 

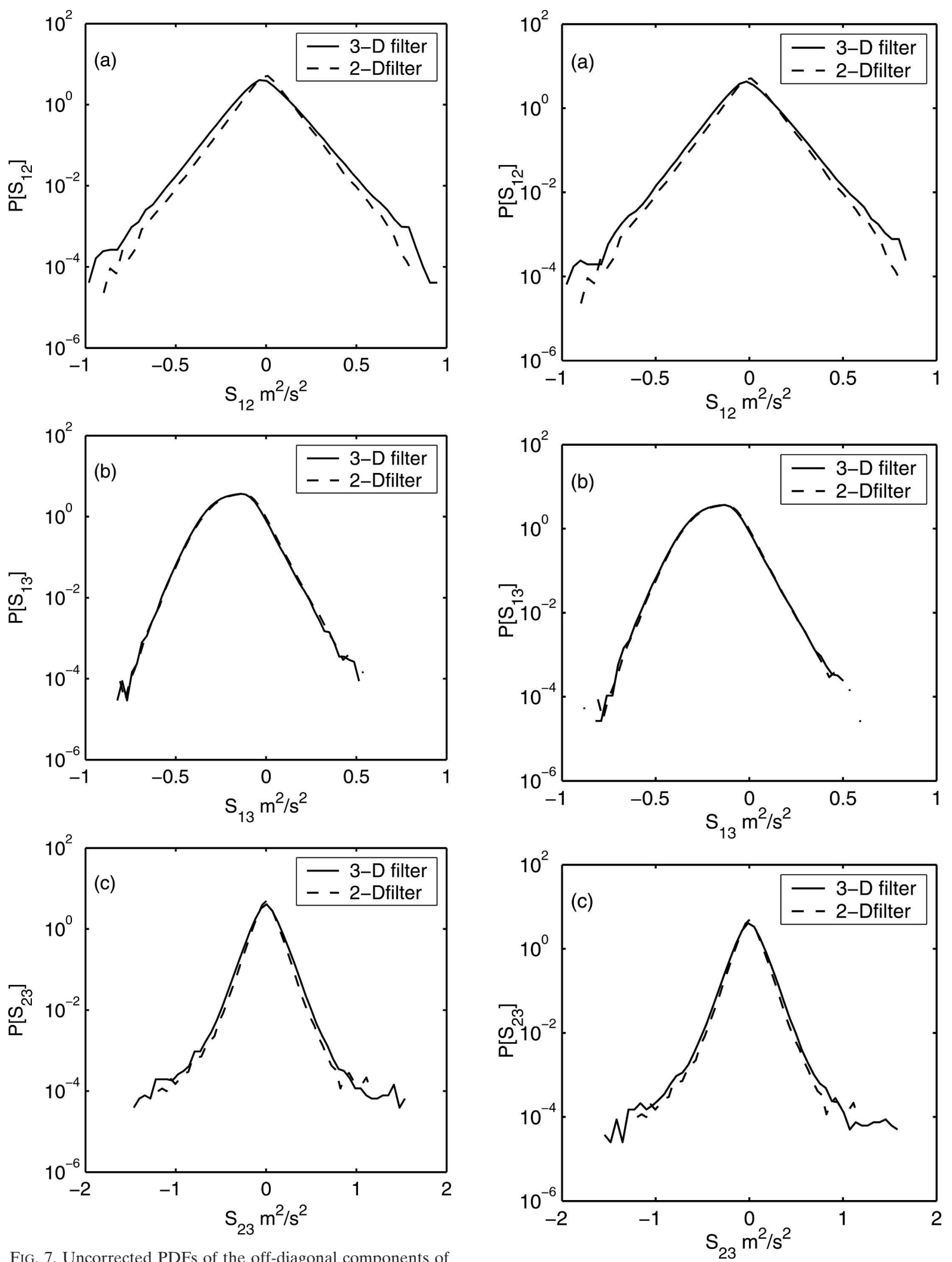

FIG. 7. Uncorrected PDFs of the off-diagonal components of the filtered strain rate.

FIG. 8. PDFs of the off-diagonal components of the filtered strain rate when $\Delta_{3-D}=0.84 \Delta_{2-D}$. 


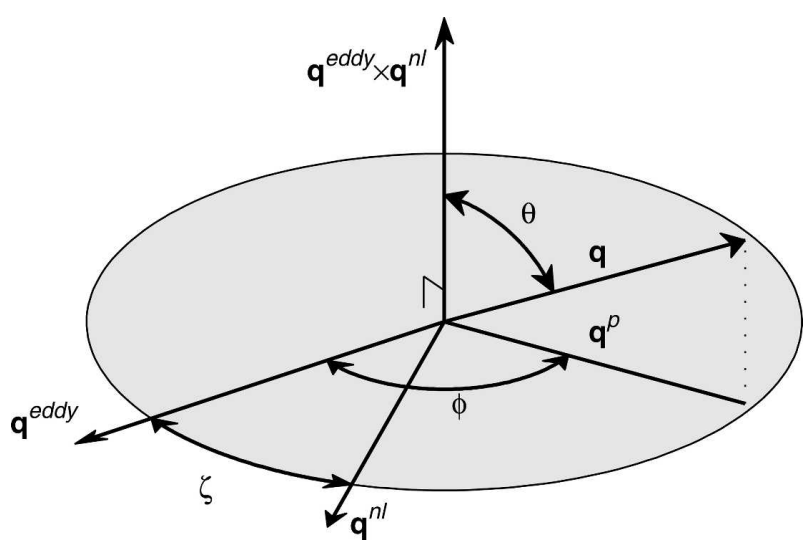

FIG. 9. A sketch of the geometry used to define the span of the mixed model and investigate relative orientations of the SGS models for the heat flux (from Higgins et al. 2004).

tive to the model-defined coordinates. In addition, a single PDF of $\zeta\left[\mathbf{q}^{\text {eddy }} \leftrightarrow \mathbf{q}^{\text {nl }}\right]$ is shown at the bottom of each plot to characterize the alignment of the tensor eddy diffusivity vector with respect to the eddydiffusion vector. A full description of this analysis technique is presented in Higgins et al. (2004).

Data from unstable periods are presented in Fig. 10. In Fig. 10b, the case in which three-dimensional filtering is used, the alignment is more pronounced (larger bright white area) than in Fig. 10a (two-dimensional filtering). However in both cases, the alignment is centralized on the plane defined by the mixed model span, and in both cases the most likely orientation of the SGS heat flux is between the nonlinear and eddy viscosity models. The main difference between Fig. 10a and Fig. $10 \mathrm{~b}$ is in the location of the peak probability. In Fig. $10 \mathrm{a}$, the case with two-dimensional filtered data, the most likely position of the measured heat flux is $\sim 54^{\circ}$ from the eddy viscosity model, $\left(\mathbf{q}^{\text {eddy }}\right)$. In the case of three-dimensionally filtered data (Fig. 10b), the more likely heat flux position is $\sim 70^{\circ}$ from the eddy viscosity model, $\mathbf{q}^{\text {eddy }}$, and only $\sim 20^{\circ}$ from the nonlinear model, $\mathbf{q}^{\mathrm{nl}}$. These observations (for the two-dimensional case) are consistent with previously reported alignments presented by Higgins et al. (2004).

In Figs. 11 and 12 there are striking differences between the alignment seen when two- (shown in Figs. 11a, 12a) or three-dimensional (shown in Figs. 11b, $12 \mathrm{~b}$ ) filterings are used for neutral and stable atmospheric stability, respectively. In all cases the alignment is localized in the plane defined by the mixed model span, but the three-dimensional filtered results consistently show strong alignment with the nonlinear model, $\mathbf{q}^{\mathrm{nl}}$, alone. The results obtained from two-dimensional filtering, however, are aligned with neither the eddy viscosity nor the nonlinear model. Here, the two-di- mensional filtered results are consistent with the structure of previously reported alignments (Higgins et al. 2004), but the location of the peak in probability is different. A discussion on how to "correct" the twodimensional filtered results will be presented in the next section on tensor alignments.

\section{Tensor alignments}

To further investigate the differences that arise between results obtained from two- and threedimensional filterings, the alignment between the SGS stress tensor and the filtered strain rate tensor is considered. The filtered strain rate and the SGS stress tensors are (by construction) symmetric and traceless; thus, the eigenvectors of the tensor are both real and orthogonal (i.e., they form a basis of three-dimensional space). Characterization of the full tensor-tensor alignment (e.g., SGS stress and strain rate tensor) requires the specification of three unique angles. The analysis is explained in detail in Tao et al. (2002) and Higgins et al. (2003), and presented briefly here. The extensive eigendirection of the SGS stress, $\boldsymbol{\alpha}_{-\tau}$, is expressed in spherical coordinates relative to the strain rate eigendirections-thus specifying two of the three unique angles $\left(\theta=\cos ^{-1}\left(\left|\boldsymbol{\alpha}_{\tilde{\mathbf{S}}} \cdot \boldsymbol{\alpha}_{-\tau}\right| /\left|\boldsymbol{\alpha}_{\tilde{\mathbf{S}}} \| \boldsymbol{\alpha}_{-\tau}\right|\right)\right.$, and $\phi=\cos ^{-1}\left(\mid \boldsymbol{\beta}_{\tilde{\mathbf{S}}} \cdot\right.$ $\left.\left.\left[\boldsymbol{\alpha}_{-\tau}-\left(\boldsymbol{\alpha}_{\tilde{\mathbf{S}}} \cdot \boldsymbol{\alpha}_{-\tau}\right) \boldsymbol{\alpha}_{\tilde{\mathbf{S}}}\right] / /\left[\boldsymbol{\alpha}_{-\tau}-\left(\boldsymbol{\alpha}_{\tilde{\mathbf{S}}} \cdot \boldsymbol{\alpha}_{-\tau}\right) \boldsymbol{\alpha}_{\tilde{\mathbf{S}}}\right] \| \boldsymbol{\beta}_{\tilde{\mathbf{S}}} \mid\right)\right)$, but the orientation of the SGS stress relative to the filtered strain rate has not been fixed completely since the compressive, $\boldsymbol{\gamma}_{-\tau}$, and intermediate, $\boldsymbol{\beta}_{-\tau}$, eigendirections are free to rotate in a plane perpendicular to $\boldsymbol{\alpha}_{-\tau}$ (see Fig. 13). The compressive eigendirection of the filtered strain rate, $\gamma_{\tilde{\mathbf{S}}}$, is projected into the plane perpendicular to the fixed SGS stress eigendirection, $\boldsymbol{\alpha}_{-\tau}$. This projection now lies in the plane spanned by two eigendirections of the SGS stress $\boldsymbol{\gamma}_{-\boldsymbol{\tau}}$ and $\boldsymbol{\beta}_{-\tau}$, and its angle, $\zeta=\cos ^{-1}\left(\left|\gamma_{-\tau} \cdot\left[\gamma_{\tilde{\mathbf{S}}}-\left(\gamma_{\tilde{\mathbf{S}}} \cdot \boldsymbol{\alpha}_{-\tau}\right) \boldsymbol{\alpha}_{-\tau}\right]\right| /\left|\boldsymbol{\gamma}_{-\tau} \|\right|\left[\gamma_{\tilde{\mathbf{S}}}-\left(\boldsymbol{\gamma}_{\tilde{\mathbf{S}}}\right.\right.\right.$ - $\left.\left.\left.\boldsymbol{\alpha}_{-\tau}\right) \boldsymbol{\alpha}_{-\tau}\right] \mid\right)$, relative to the compressive direction of the SGS stress, $\gamma_{-\tau}$, will fix the relative position of the two tensors. A sketch of the geometry used to define these angles is presented in Fig. 13.

In Figs. 14a-f, respectively, the alignment between the filtered strain rate and SGS stress is shown for unstable three-dimensional filtered data, unstable twodimensional filtered data, near-neutral three-dimensional filtered data, near-neutral two-dimensional filtered data, stable three-dimensional filtered data, and stable two-dimensional filtered data. The tensor alignment is similar in structure between three-dimensionally filtered (Fig. 14a) and two-dimensionally filtered (Fig. 14b) data for the case of unstable atmospheric stability. The three-dimensionally filtered (Fig. 14a) data show an increased probability of alignment 

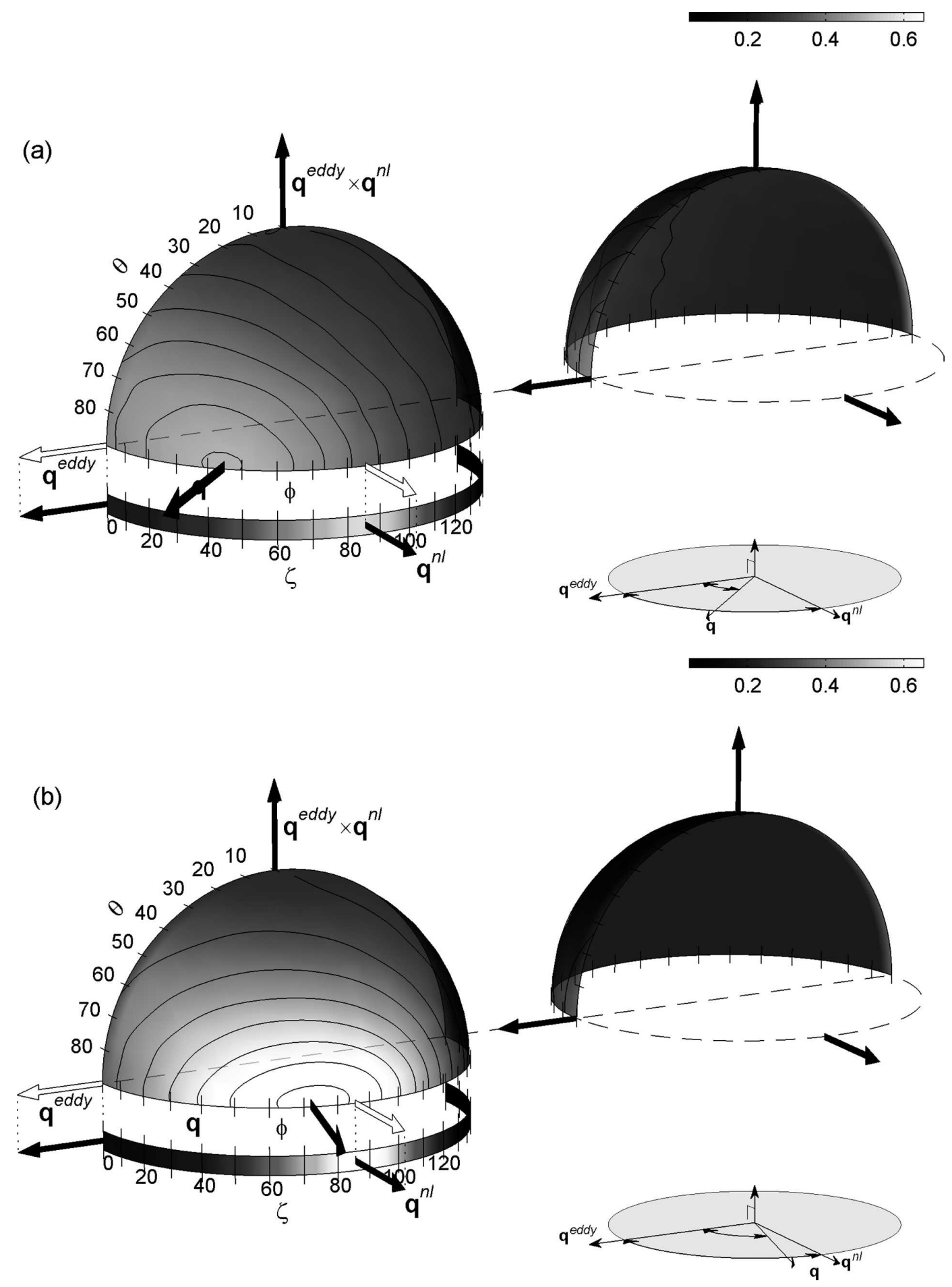

FIG. 10. Heat flux alignment PDFs under unstable atmospheric stability: results when (a) two- and (b) three-dimensional filterings are used.

(darker red areas); however, the most likely alignment pattern (i.e., the location of areas of increased probability) is similar. In the near neutral case, the threedimensionally filtered (Fig. 14c) data show an increased probability of alignment (darker red areas); however, the most likely alignment pattern (i.e., the location of areas of increased probability) is similar. In both cases of atmospheric stability (unstable and near neutral), the 


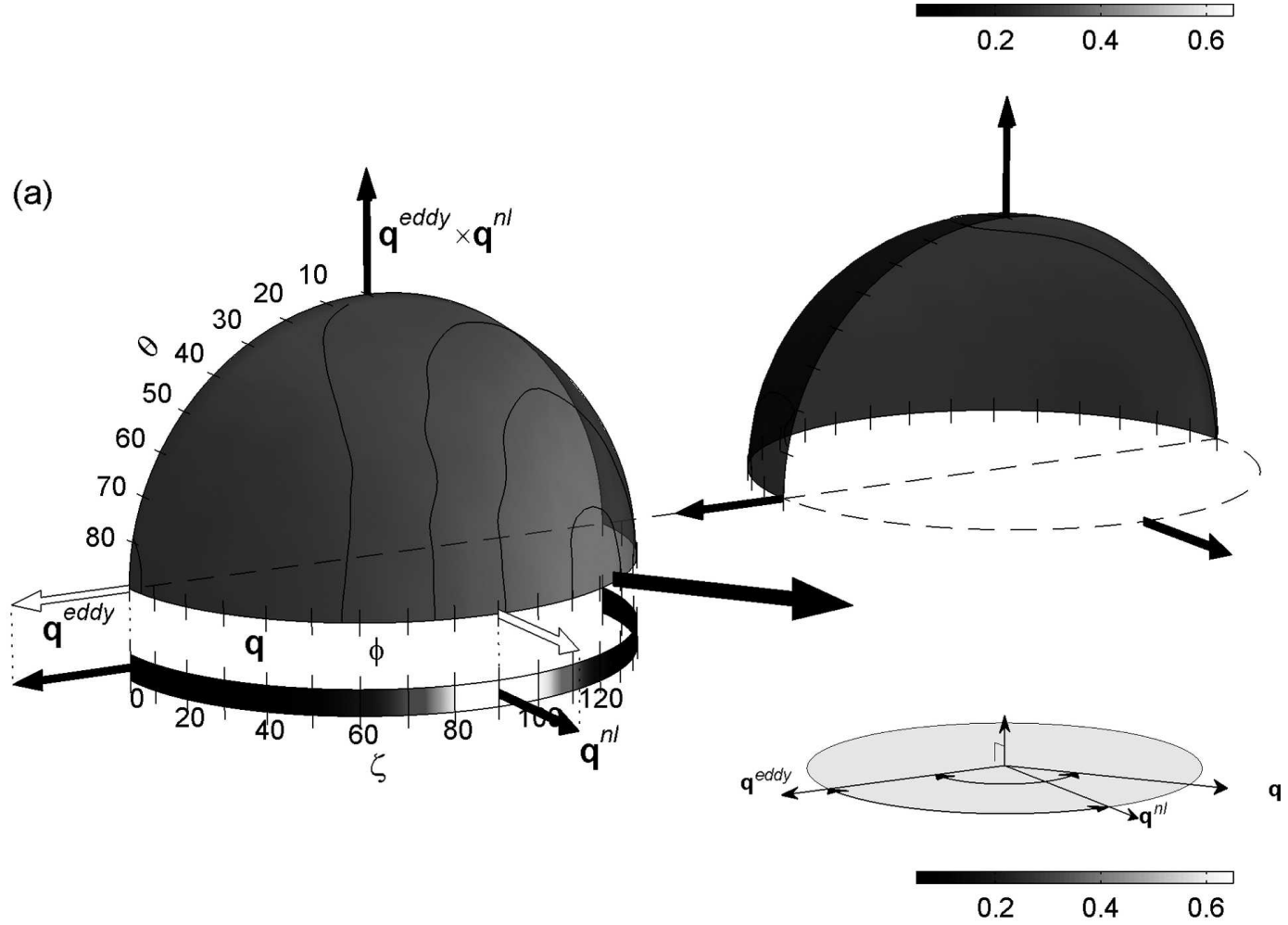

(b)
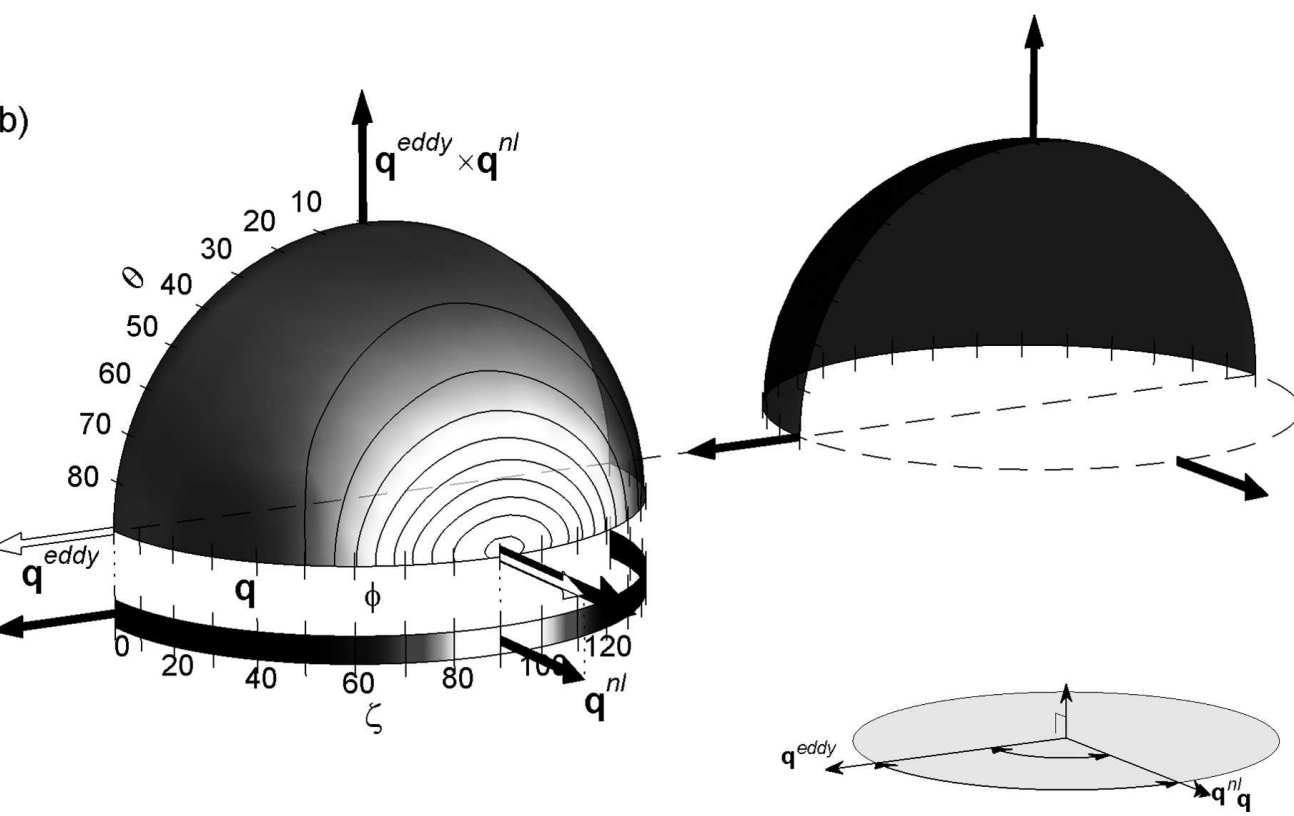

FIG. 11. Heat flux alignment PDFs under neutral atmospheric stability: results when (a) two- and (b) three-dimensional filterings are used.

alignment trends shown are consistent with previously reported results of Tao et al. (2002) and Higgins et al. (2003).

Major differences arise when we compare the results obtained from three-dimensional filtering (Fig. 14e) and two-dimensional filtering (Fig. 14f) in the case of stable atmospheric stability. The three-dimensionally filtered data exhibit a similar alignment trend seen in 


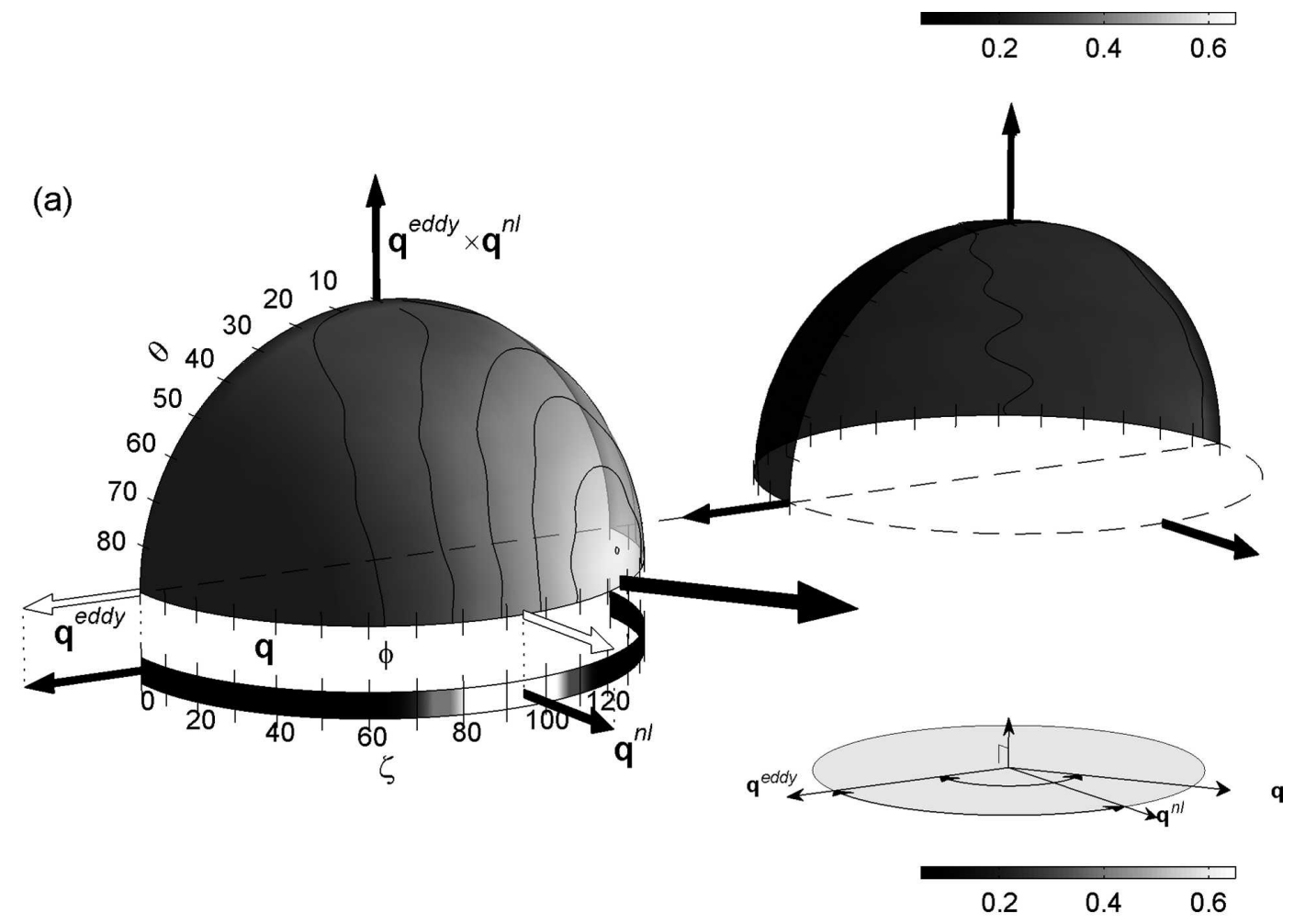

(b)
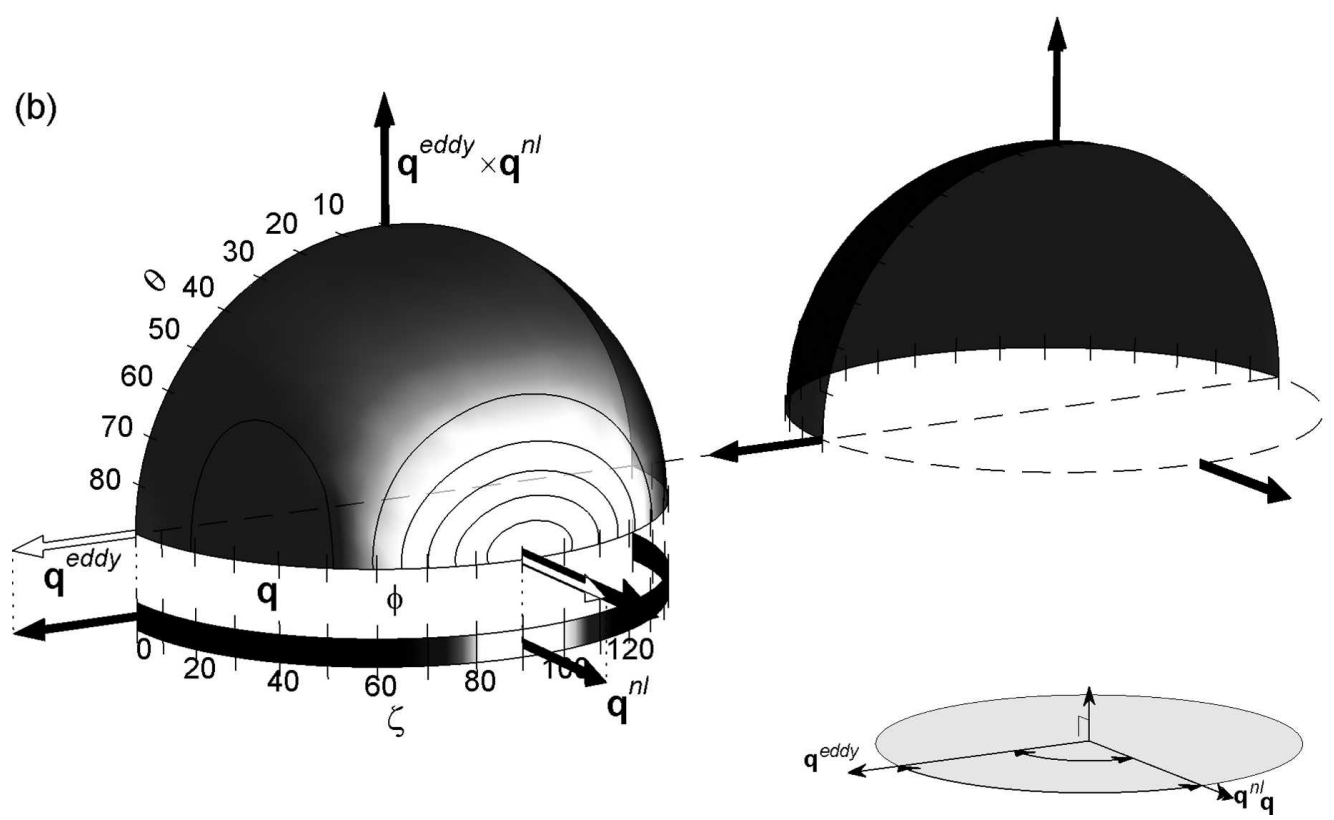

FIG. 12. Heat flux alignment PDFs under stable atmospheric stability: results when (a) two- and (b) three-dimensional filterings are used.

the previously discussed cases of near-neutral (Fig. 14c) and unstable (Fig. 14a) atmospheric stability. However, the alignment pattern given by the two-dimensionally filtered data (Fig. 14f) is strikingly different than the three-dimensional filtered alignment pattern, and in this case using a two-dimensional surrogate for threedimensional filtering would yield misleading results. This is the first time that the tensor alignment trend for 


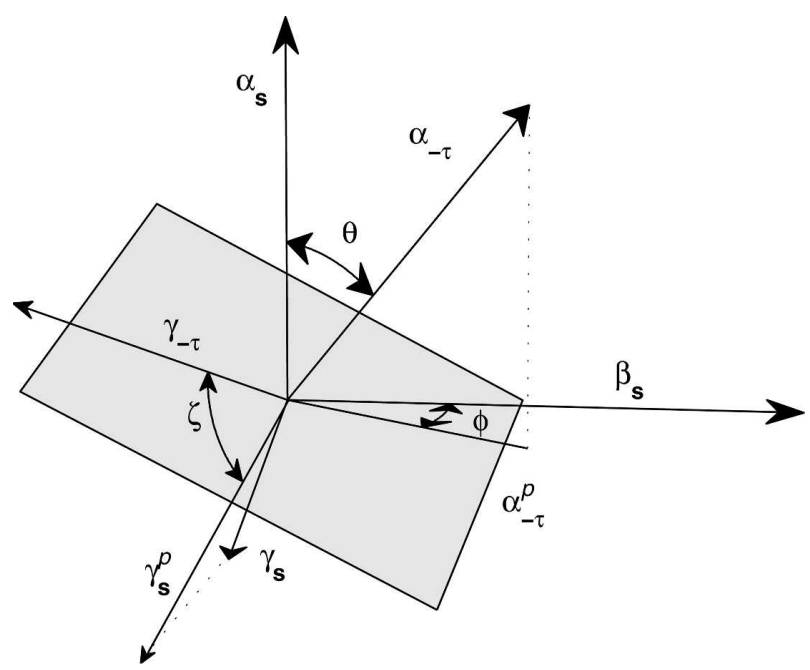

FIG. 13. Sketch of the geometry used to define the relative orientation of the SGS stress and filtered strain rate (from Higgins et al. 2003).

the case of stable atmospheric stability has been reported.

\section{Recommendations}

It is preferable to apply three-dimensional filtering whenever possible, but when data or resources are not available, one can obtain accurate statistics of the SGS stress and filtered strain rate components from twodimensional filtering by interpreting the filter size as equivalent to a three-dimensional filter at scale $\Delta_{3-D}=$ $0.84 \Delta_{2-D}$, as seen in the first section of this paper. When performing more detailed analyses, such as the alignment analyses presented above, two-dimensional filtering can still give reliable results for the case of unstable atmospheric stability. Poor agreement between two-dimensionally filtered and three-dimensionally filtered results arises in the cases of nearneutral and stable atmospheric stabilities in the heat flux alignment analyses. Further discrepancies are shown for the case of stable atmospheric stability with the tensor alignment analysis. It is possible, however, to get accurate enough results while still using twodimensional filtering in both the heat flux and tensor alignment analyses. As explained in the previous sections, in all the previous analyses the data are filtered in two dimensions along horizontal planes. The strength of the vertical gradient in the stable case (shown in Fig. 2d) leads one to surmise that it may be better to filter along vertical planes for this case. If the data from the SGS 2002 dataset (under stable atmospheric stability) are filtered along vertical planes in the vertical and streamwise directions, the two-dimensional filtered results show a great improvement. Figure 15 shows the results from the tensor alignment analysis with twodimensional filtering done in the vertical direction. Recall, Fig. 14e is the result of the tensor alignment analysis when three-dimensional filtering is used. Now both two- and three-dimensional filterings give similar results in both magnitude and structure. Note that all previous experiments and analyses (Tong et al. 1999, 1998; Porté-Agel et al. 1998, 2000a,b, 2001a,b; Higgins et al. 2003; Horst et al. 2004; Kleissl et al. 2003) are performed with the two-dimensional horizontal filtering technique.

Two-dimensional filtering in the vertical (wall normal) direction also improves the match between threedimensionally filtered results and two-dimensionally filtered results for the heat flux analysis. Again in the stable case there were large discrepancies between the three- and two-dimensional filtered results (Figs. 12a and 12b). If this analysis is redone with the proposed two-dimensional filtering in the vertical and streamwise directions, there is a marked improvement in the comparison of the results. This is shown in Fig. 16. The results obtained from two-dimensional filtering in the vertical-streamwise directions are shown to be comparable with the three-dimensional results shown in Fig. $12 \mathrm{~b}$. This result suggests that experiment design (measurement array geometry) should be carefully considered if a two-dimensional surrogate filter is to be used. If stably stratified flows are of primary interest, vertical array geometry, and the consequent vertical filtering, would produce better results.

\section{Summary}

Field experiments performed with arrays of sonic anemometers to measure small-scale spatial aspects of atmospheric turbulence in the context of large eddy simulation have been important for understanding SGS stresses, heat fluxes, and their parameterizations in the lower atmosphere. To date, most experiments are based on the assumption that two-dimensional filtering is an appropriate surrogate for the three-dimensional filtering used to define the SGS quantities. We have shown that the components of the SGS stress computed with two-dimensional filtering have greater intermittency than those calculated with three-dimensional filtering. Through spectral and statistical analysis we also show that this difference in intermittency can be reduced or eliminated if the three-dimensional filter scale is interpreted properly.

In another detailed analysis of the alignments of the 

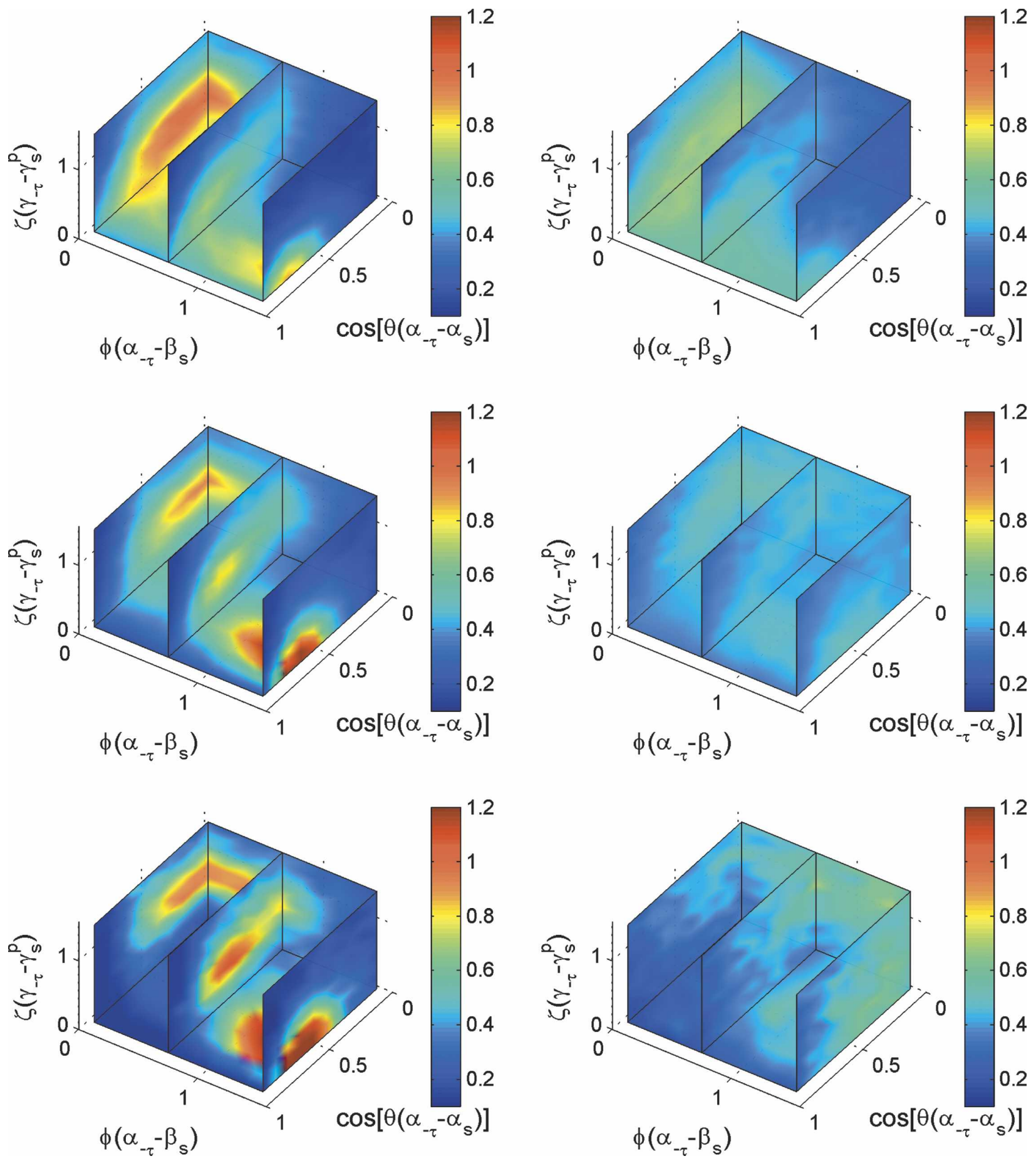

FIG. 14. Tensor alignment PDFs: (a) unstable and 3D filter, (b) unstable and 2D filter, (c) near-neutral and 3D filter, (d) near-neutral and 2D filter, (e) stable and 3D filter, and (f) stable and 2D filter.

SGS heat flux and the tensor alignment between the SGS stress and the filtered strain rate, we show that two-dimensional filtering yields satisfactory results in the case of unstable atmospheric stability, but that in the case of stable atmospheric stability, two- dimensional filtering along horizontal planes yields results that are significantly different from those of threedimensional filtering. When two-dimensional filtering is unavoidable, better results can be obtained by filtering in two dimensions along vertical planes, that is, in 


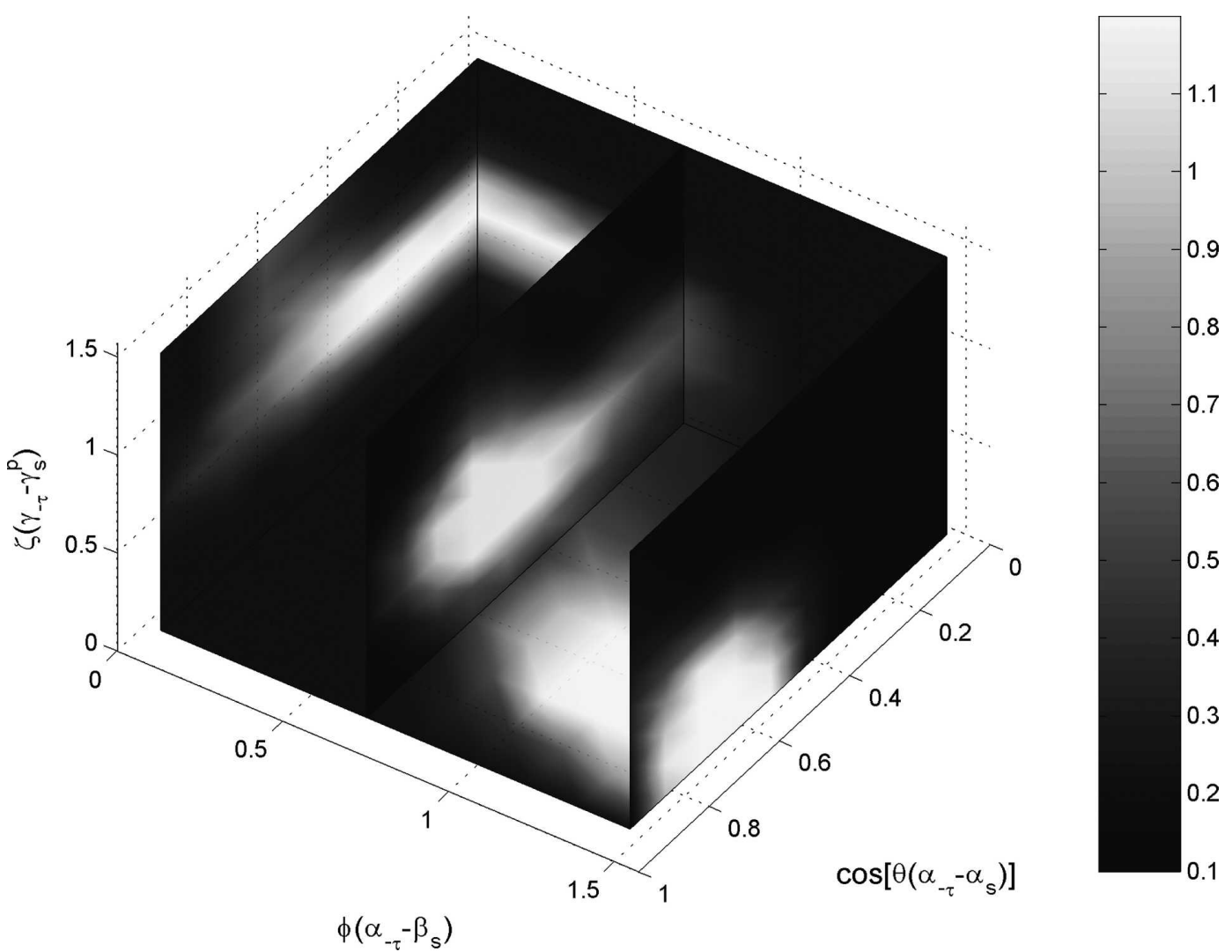

FIG. 15. Tensor alignment analysis for stable data results computed with two-dimensional vertical filtering.

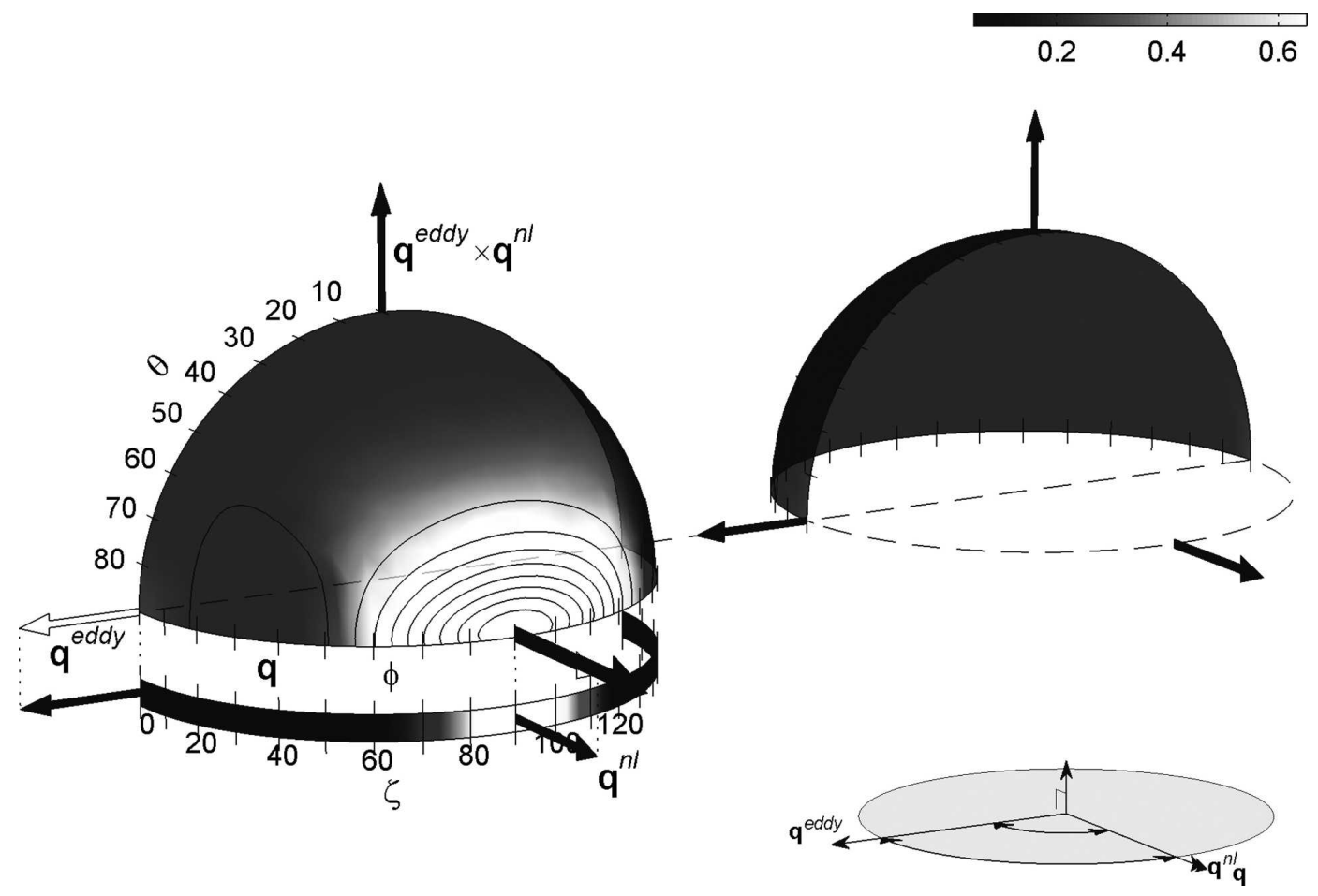

FIG. 16. Vector alignment analysis for stable data results computed with two-dimensional vertical filtering. 
the wall normal-streamwise plane that samples the contribution from the mean shear and temperature gradient.

Acknowledgments. We gratefully acknowledge the field support and cooperation of Prof. Joe Klewicki of the University of Utah, who made the field campaign possible, and the support of colleagues Jan Kleissl, Vijayant Kumar, Fernando Porté-Agel, and others from the Dugway proving ground. Partial funding is provided by NSF-ATM 0130766.

\section{REFERENCES}

Bardina, J., J. H. Ferziger, and W. C. Reynolds, 1980: Improved subgrid scale models for large eddy simulation. American Institute of Aeronautics Astronautics Paper 80-1357.

Borue, V., and S. A. Orszag, 1998: Local energy flux and subgridscale statistics in three-dimensional turbulence. J. Fluid Mech., 366, 1-31.

De Stefano, G., and O. V. Vasilyev, 2002: Sharp cut-off vs. smooth filtering in LES. Phys. Fluids, 14, 362-369.

Higgins, C. W., M. B. Parlange, and C. Meneveau, 2003: Alignment trends of velocity gradients and subgrid-scale fluxes in the turbulent atmospheric boundary layer. Bound.-Layer Meteor., 109, 59-83.

,-- , and -2004 : The heat flux and the temperature gradient in the lower atmosphere. Geophys. Res. Lett., 31, L22105, doi:10.1029/2004GL020053.

Horst, T. W., J. Kleissl, D. H. Lenschow, C. Meneveau, C.-H. Moeng, M. B. Parlange, P. P. Sullivan, and J. C. Weil, 2004: HATS: Field observations to obtain spatially filtered turbulence fields from transverse arrays of sonic anemometers in the atmospheric surface layer. J. Atmos. Sci., 61, 1566-1581.

Kleissl, J., C. Meneveau, and M. B. Parlange, 2003: On the magnitude and variability of subgrid-scale eddy-diffusion coefficients in the atmospheric boundary layer. J. Atmos. Sci., 60, 2372-2388.

, M. B. Parlange, and C. Meneveau, 2004: Field experimental study of dynamic Smagorinsky models in the atmospheric surface layer. J. Atmos. Sci., 61, 2296-2307.
Leonard, A., 1974: Energy cascade in large-eddy simulations of turbulent fluid flows. Advances in Geophysics, Vol. 18, Academic Press, 237-248.

_ 1997: Large-eddy simulation of chaotic convection and beyond. American Institute of Aeronautics and Astronautics Paper 97-0204, 1-8.

Piomelli, U., J. Ferziger, and P. Monin, 1988: Model consistency in large eddy simulation of turbulent channel flows, Phys. Fluids, 31, 1884-1891.

Porté-Agel, F., C. Meneveau, and M. B. Parlange, 1998: Some basic properties of the surrogate subgrid-scale heat flux in the atmospheric boundary layer. Bound.-Layer Meteor., 88, 425444.

,-- , and - , 2000a: A scale dependent dynamic model for large eddy simulation: Application to a neutral atmospheric boundary layer. J. Fluid Mech., 415, 261-284.

-, M. B. Parlange, C. Meneveau, W. E. Eichinger, and M. Pahlow, 2000b: Subgrid-scale dissipation in the atmospheric surface layer: Effects of stability and filter dimension. J. Hydrometeor., 1, 75-87.

— M. Pahlow, C. Meneveau, and M. B. Parlange, 2001a: Atmospheric stability effect on subgrid scale physics for largeeddy simulation. Adv. Water Resour., 24, 1085-1102.

— M. B. Parlange, C. Meneveau, and W. E. Eichinger, 2001b: A priori field study of the subgrid-scale heat fluxes and dissipation in the atmospheric surface layer. J. Atmos. Sci., 58, 2673-2697.

Smagorinsky, J., 1963: General circulation experiments with primitive equations. Mon. Wea. Rev., 91, 99-164.

Sullivan, P. P., T. W. Horst, D. H. Lenschow, C. H. Moeng, and J. C. Weil, 2003: Structure of subfilter-scale fluxes in the atmospheric surface layer with application to large-eddy simulation modeling. J. Fluid Mech., 482, 101-139.

Tao, B., J. Katz, and C. Meneveau, 2002: Statistical geometry of subgrid-scale stresses determined from holographic particle image velocimetry measurements. J. Fluid Mech., 457, 35-78.

Tong, C. N., J. C. Wyngaard, S. Khanna, and J. G. Brasseur, 1998: Resolvable- and subgrid-scale measurement in the atmospheric surface layer: Technique and issues. J. Atmos. Sci., 55, 3114-3126.

— - _ and J. G. Brasseur, 1999: Experimental study of the subgrid-scale stress in the atmospheric surface layer. J. Atmos. Sci., 56, 2277-2292. 